\title{
A Combinatorial Property of Points and Balls, a Colored Version
}

\author{
Maria N. Prodromou
}

Received: 14 September 2004 / Revised: 20 October 2006 /

Published online: 2 September 2007

(C) Springer Science+Business Media, LLC 2007

\begin{abstract}
Any finite set $X \subset \mathbb{R}^{d}$ colored with $\left\lfloor\frac{d+3}{2}\right\rfloor$ colors, contains a rainbow subset $Y \subset X$, such that any ball that contains $Y$ contains a positive fraction of the points of $X$. The bound on the number of colors is tight.
\end{abstract}

\section{Introduction}

Any finite set $X$ of points in the plane in general position, contains two points such that, any disc that contains them, necessarily contains a positive fraction of the points of $X$. This result was proved by Neumann-Lara and Urrutia in [6]. They proved that any such set contains two points such that any disc that contains them, will contain at least $\lceil(n-2) / 60\rceil$ points of $X$, where $n$ is the number of points in $X$. Hayward in [3] improved the bound to $\left\lceil\frac{5}{84}(n-2)\right\rceil$. Hayward et al. in [4] showed that if $\Pi(n)$ is the largest number, such that for every $n$-point set $X$ on the plane, there exist two points $x, y \in X$ with this property, then $\lfloor n / 27\rfloor+2 \leq \Pi(n) \leq\lceil n / 4\rceil+1$.

The planar case was later generalized by Bárány et al. in [2] to the case of $X \subset \mathbb{R}^{d}$. They proved that any $n$-point set $X \subset \mathbb{R}^{d}$ in general position contains a subset $A$ with $|A|=\left\lfloor\frac{1}{2}(d+3)\right\rfloor$ such that, any ball that contains it, contains $c(d)|X|$ points, where the constant $c(d)>0$ is at least $(m !(d-m-1) !) / d !$ and $m=\left\lfloor\frac{1}{2}(d+3)\right\rfloor$. They also showed that the bound on the number of points of $A$ is best possible.

Bárány and Larman in [1] generalized this result from the case of Euclidean balls, to the case of ellipsoids and more generally, quadrics in $\mathbb{R}^{d}$. They show that there is a constant $c(d)>0$ such that any $n$-point set $X \subset \mathbb{R}^{d}$ contains a subset $Y$, with $|Y|=$

This work was done while the author was visiting the Mathematical Sciences Research Institute, Berkeley. The author was supported by the UCL Graduate School Research Grant, \#1.03/04.RP.EMB. 
$\left\lfloor\frac{1}{4} d(d+3)\right\rfloor$, with the property that any quadric that contains $Y$ contains $c(d)|X|$ points of $X$. In this case $c(d) \geq m^{-1} 2^{-(m+1)}$, where $m=(d+1)(d+2) / 2$. Again it is shown that the bound on the number of points in $Y$ is best possible.

Here we consider the colored case. Throughout the paper, $k=\left\lfloor\frac{d+3}{2}\right\rfloor$. The system of sets $C_{1}, C_{2}, \ldots, C_{k} \subset \mathbb{R}^{d}$ will be $k$ disjoint, finite sets with $\left|C_{i}\right|=s, s \geq 3$, for all $i=1, \ldots, k$. Their union $\bigcup_{i=1}^{k} C_{i}$ will be denoted by $X$ and $n$ will be the cardinality of $X$. A set $Y \subset X$ is a transversal for the system $C_{1}, \ldots, C_{k}$, if $\left|Y \cap C_{i}\right|=1$, for all $i=1, \ldots, k$. Let $\mathcal{B} \subset \mathcal{P}\left(\mathbb{R}^{d}\right)$ denote the set of closed Euclidean balls in $\mathbb{R}^{d}$. Our main result is the following.

Theorem 1.1 For any $d \geq 1$, there is a constant $c(d)>0$ such that for any system $C_{1}, C_{2}, \ldots, C_{k} \subset \mathbb{R}^{d}$, there is a transversal $Y \subset X$, such that for any $B \in \mathcal{B}$ with $Y \subset B$, it follows that $|B \cap X| \geq c(d)|X|$.

The constant here is $c(d)=\frac{1}{2 k 3^{k}}$. If the sets $C_{1}, \ldots, C_{k}$ are thought of as colors, then Theorem 1.1 says that there is a rainbow set $Y \subset X$ such that any ball that contains it, contains a positive fraction of the points of $X$.

The main tool for proving Theorem 1.1 is Lemma 1.2 below, the proof of which uses the following beautiful result of Vrećica and Živaljević from [7] (see also [5], or [8] and [9] for an exposition of the two results).

Theorem 1 (Vrećica and Živaljević) Let $C_{1}, C_{2}, \ldots, C_{k} \subset \mathbb{R}^{d}$ be finite sets, with $\left|C_{i}\right|=2 p-1, p(d-k+1) \leq d$ where $p$ is a prime. Then there are $A_{1}, \ldots, A_{p} \subset \mathbb{R}^{d}$ with $A_{i} \cap A_{j}=\varnothing$, for $i \neq j,\left|A_{i} \cap C_{j}\right|=1$, for all $i, j$ and $\bigcap_{i=1}^{p} \operatorname{conv} A_{i} \neq \varnothing$.

We will apply the $p=2$ case of this result in the proof of Lemma 1.2. Then $k$ can be taken to be $\left\lfloor\frac{d+3}{2}\right\rfloor$.

Lemma 1.2 Let $X \subset \mathbb{R}^{d}$ be a finite set, $X=C_{1} \cup C_{2} \cup \cdots \cup C_{k}$, with $C_{i} \cap C_{j}=\varnothing$, for all $i \neq j$, where $k=\left\lfloor\frac{d+3}{2}\right\rfloor$ and $\left|C_{i}\right|=3$. Then, there is $Y \subset X$ with $|Y|=k$, $\left|Y \cap C_{i}\right|=1$, for all $i=1, \ldots, k$, such that for any $B \in \mathcal{B}$ with $Y \subset B$, we have $B \cap(X \backslash Y) \neq \varnothing$.

In the next two Theorems, the sets $C_{i}, i=1, \ldots, k$ can be finite or infinite. The following Theorem shows that the number of color classes in Theorem 1.1 is best possible.

Theorem 1.3 For any $d \geq 1$ there is a system $C_{1}, C_{2}, \ldots, C_{k-1} \subset \mathbb{R}^{d}$, such that for any transversal $Y \subset X$, there is $B \in \mathcal{B}$ with $Y \subset B$ and $B \cap X=Y$.

Though we know from Theorem 1.1 that we can always find a rainbow subset $Y \subset X$ such that any $B \in \mathcal{B}$ that contains it, will contain a positive fraction of the points of $X$, we can't hope that this fraction will come from many of the color classes. In fact, all points (but the points of $Y$ ) might come from a single color class.

Theorem 1.4 For any $d \geq 1$ there is a system $C_{1}, C_{2}, \ldots, C_{k}$, such that for any transversal $Y \subset X$, there is a ball $B \in \mathcal{B}$ such that $Y \subset B,\left|B \cap C_{i}\right|=1$, for all $i \in\{1, \ldots, k\} \backslash\left\{i_{0}\right\}$ and $\left|B \cap C_{i_{0}}\right| \geq c(d)|X|$, for some $i_{0} \in\{1, \ldots, k\}$. 
In Sect. 5, we show that the result of Theorem 1.1 also holds for more general families $\mathcal{F} \subset \mathcal{P}\left(\mathbb{R}^{d}\right)$, where $F \in \mathcal{F}$ is given by

$$
F=\left\{x \in \mathbb{R}^{d}: \sum_{i=1}^{m} \alpha_{i} f_{i}(x) \leq 0\right\},
$$

for $f_{i}: \mathbb{R}^{d} \rightarrow \mathbb{R}, i=1, \ldots, m$ and $\alpha_{i} \in \mathbb{R}$ not all zero.

\section{Proof of Theorem 1.1}

We start with the proof of Lemma 1.2. In the proof we use the notation $x^{2}$ and $x y$ for the squared norm of $x$ and the scalar product of $x, y$ respectively.

Proof From Theorem 1, for the case $p=2$ we have $A_{1}, A_{2} \subset X$, with $A_{1} \cap A_{2}=\varnothing$, $\left|A_{i} \cap C_{j}\right|=1$, for all $i, j$ and $\operatorname{conv} A_{1} \cap \operatorname{conv} A_{2} \neq \varnothing$. Suppose there is no subset $Y$ of $X$ that satisfies the statement of the Lemma. In particular, the subsets $A_{1}, A_{2}$ do not satisfy it. Then, there are $B_{1}, B_{2} \in \mathcal{B}$ such that $A_{1} \subset B_{1}, A_{2} \subset B_{2}$ and $B_{1} \cap$ $X \backslash A_{1}=\varnothing$ and $B_{2} \cap X \backslash A_{2}=\varnothing$. In particular, $B_{1} \cap A_{2}=\varnothing$ and $B_{2} \cap A_{1}=\varnothing$. Write

$B_{1}=\left\{x \in \mathbb{R}^{d}:\left(x-c_{1}\right)^{2} \leq r_{1}^{2}\right\}$ and $B_{2}=\left\{x \in \mathbb{R}^{d}:\left(x-c_{2}\right)^{2} \leq r_{2}^{2}\right\}$, for some $c_{1}, c_{2} \in \mathbb{R}^{d}, r_{1}, r_{2}>0$. Then, all of the following hold:

$$
\begin{array}{lll}
x^{2}-2 c_{1} x+c_{1}^{2} \leq r_{1}^{2} \quad \text { and } \quad x^{2}-2 c_{2} x+c_{2}^{2}>r_{2}^{2}, & \forall x \in A_{1}, \\
y^{2}-2 c_{2} y+c_{2}^{2} \leq r_{2}^{2} & \text { and } \quad y^{2}-2 c_{1} y+c_{1}^{2}>r_{1}^{2}, \quad \forall y \in A_{2},
\end{array}
$$

or,

$$
2\left(c_{2}-c_{1}\right) x<\left(r_{1}^{2}-r_{2}^{2}\right)+\left(c_{2}^{2}-c_{1}^{2}\right), \quad \forall x \in A_{1}
$$

and

$$
2\left(c_{2}-c_{1}\right) y>\left(r_{1}^{2}-r_{2}^{2}\right)+\left(c_{2}^{2}-c_{1}^{2}\right), \quad \forall y \in A_{2} .
$$

Let $z \in \operatorname{conv} A_{1} \cap \operatorname{conv} A_{2}$. Then

$$
z=\sum_{x \in A_{1}} \lambda(x) x=\sum_{y \in A_{2}} \mu(y) y,
$$

for some $0 \leq \lambda(x), \mu(y) \leq 1$, with $\sum_{x \in A_{1}} \lambda(x)=\sum_{y \in A_{2}} \mu(y)=1$.

Multiply (1) by $\lambda(x) \geq 0, x \in A_{1}$ and sum over all $x \in A_{1}$ and (2) by $\mu(y) \geq 0$, $y \in A_{2}$ and sum over all $y \in A_{2}$. Then,

$$
\left(r_{1}^{2}-r_{2}^{2}\right)+\left(c_{2}^{2}-c_{1}^{2}\right)<2\left(c_{2}-c_{1}\right) z<\left(r_{1}^{2}-r_{2}^{2}\right)+\left(c_{2}^{2}-c_{1}^{2}\right) .
$$

This contradiction completes the proof.

Lemma 1.2 says that if the assertion is false for both $A_{1}, A_{2}$, then a point in the intersection of their convex hulls, will lie in both open half spaces of the hyperplane defined by the intersection of the boundaries of $B_{1}$ and $B_{2}$. 
Proof of Theorem 1.1 Since for every $Z \subset X$ with $\left|Z \cap C_{i}\right|=3$ there is a $Y$ which satisfies the properties of Lemma 1.2, there is a family $\mathcal{Z} \subset \mathcal{P}(X)$ and $Y \subset X$, with $\left|Y \cap C_{i}\right|=1$, for $i=1, \ldots, k$, such that $Y$ belongs to every $Z \in \mathcal{Z}$ and

$$
|\mathcal{Z}| \geq \frac{\left(\begin{array}{l}
s \\
3
\end{array}\right)^{k}}{\left(\begin{array}{l}
s \\
1
\end{array}\right)^{k}}=\frac{(s-2)^{k}(s-1)^{k}}{6^{k}},
$$

where $\left|C_{i}\right|=s$, for all $i=1, \ldots, k, \sum_{i=1}^{k}\left|C_{i}\right|=s k=|X|$ and $k=\left\lfloor\frac{d+3}{2}\right\rfloor$. We will now obtain an upper bound on $|\mathcal{Z}|$.

Let $B \in \mathcal{B}$ be such that $Y \subset B$ and write $|B \cap X|=m$. From Lemma 1.2, each $Z \in \mathcal{Z}$ contains a point of $B \backslash Y$. So, the number of ways to extend $Y$ to $Z$, provided that $B \cap(Z \backslash Y) \neq \varnothing$ is at most

$$
\left(\begin{array}{c}
m-k \\
1
\end{array}\right)\left(\begin{array}{c}
s-1 \\
2
\end{array}\right)^{k-1}\left(\begin{array}{c}
s-2 \\
1
\end{array}\right) .
$$

The first term is the number of ways to choose a point of $Z$ from the remaining $m-k$ points in $B$. The color class to which this point belongs, will be extended by one point, which is the third term. The second term is the number of ways to extend the remaining $k-1$ color classes by two points. This is equal to

$$
(m-k) \frac{(s-2)^{k-1}(s-1)^{k-1}}{2^{k-1}}(s-2) .
$$

This is an upper bound on $|\mathcal{Z}|$. From (3) and (4) we get

$$
\frac{1}{2 \cdot 3^{k}}(s-1) \leq m-k
$$

or

$$
\frac{s k-k}{2 k 3^{k}} \leq m-k
$$

Since $s k=|X|$ and $m=|B \cap X|$ we get $|B \cap X| \geq \frac{1}{2 k 3^{k}}|X|=c(d)|X|$.

\section{Proof of Theorem 1.3}

This is very similar to Theorem 6 in [2]. Consider the moment curve $\gamma(t)=$ $\left(t, t^{2}, \ldots, t^{d}\right)$. We first deal with the case when $d$ is odd, $d=2 m-1$. Let

$$
\epsilon>t_{1}>t_{2}>\cdots>t_{m}>0
$$

be positive real numbers and $\epsilon>0$ to be specified later. We shall find constants $c_{0}, \ldots, c_{2 d}$ so that the polynomials

$$
p(t)=\left(t-c_{1}\right)^{2}+\left(t^{2}-c_{2}\right)^{2}+\cdots+\left(t^{d-1}-c_{d-1}\right)^{2}+\left(t^{d}-c_{d}\right)^{2}
$$

and

$$
q(t)=c_{0}+\left[\left(t-t_{1}\right)\left(t-t_{2}\right) \cdots\left(t-t_{m}\right)\right]^{2} r(t),
$$


where

$$
r(t)=c_{d+1}+c_{d+2} t+\cdots+c_{2 d} t^{2 m-2},
$$

are identically equal. This implies that when $t$ takes the values $t_{1}, \ldots, t_{m}$ the points $\gamma\left(t_{i}\right), i=1, \ldots, m$ belong to the surface of the ball centered at $\left(c_{1}, \ldots, c_{d}\right)$ of radius $\sqrt{c_{0}}$. It is not hard to see that the $c_{i}$ 's can be uniquely determined in the order $c_{2 d}, \ldots, c_{0}$ and are polynomial functions of $t_{1}, \ldots, t_{m}$ with constant terms given by,

$$
c_{i}= \begin{cases}0+O(\epsilon), & i \text { odd, } \\ 1 / 2+O(\epsilon), & i \in\{2, \ldots, d-1\}, i \text { even, } \\ 1+O(\epsilon), & i \in\{d+1, \ldots, 2 d\}, i \text { even }\end{cases}
$$

and finally

$$
c_{0}=\frac{m-1}{4}+O(\epsilon) .
$$

It is also easy to see that for these values of $c_{d+1}, \ldots, c_{2 d}$, the polynomial term $r(t)$ is positive when $t>0$. Choose $\epsilon>0$ so small, so that $c_{0}$ is positive. Let $C_{i}=U_{\epsilon^{\prime}}\left(\gamma\left(s_{i}\right)\right)=\left\{\gamma(t), t \in\left[s_{i}-\epsilon^{\prime}, s_{i}+\epsilon^{\prime}\right]\right\}, i=1, \ldots, m$ be sufficiently small neighborhoods of $\gamma\left(s_{i}\right)$, where $\gamma\left(s_{i}\right)$ are points taken from the part of the moment curve with $\{\gamma(t), t \in(0, \epsilon)\}$. Choose $0<\epsilon^{\prime}<\epsilon$ so small, so that the $C_{i}$ 's are disjoint. Set $X$ to be $X=\bigcup_{i=1}^{m} C_{i}$ (finite or infinite). Then, for any $Y \subset X$ with $\left|Y \cap C_{i}\right|=1$, for all $i=1, \ldots, m$, there is a ball $B \in \mathcal{B}$ centered at $\left(c_{1}, \ldots, c_{d}\right)$ of radius $\sqrt{c_{0}}$, that contains $Y$, for which $B \cap X=Y$.

The case when $d$ is even, $d=2 m$ can be dealt with similarly. In this case, the constants $c_{i}, i=1, \ldots, 2 d-1$ are not uniquely defined $\left(c_{d+1} \in \mathbb{R}\right)$. In this case, we may choose $c_{d+1}=0+O(\epsilon)$. Then the $c_{i}$ 's are given by

$$
c_{i}= \begin{cases}0+O(\epsilon), & i \text { odd, } i \in\{1, \ldots, d+1\}, \text { or } \\ & i \text { even, } i \in\{d+2, \ldots, 2 d-2\}, \\ 1 / 2+O(\epsilon), & i \text { even, } i \in\{2, \ldots, d\}, \\ 1+O(\epsilon), & i \text { odd, } i \in\{d+3, \ldots, 2 d-1\}\end{cases}
$$

and finally

$$
c_{0}=\frac{m}{4}+O(\epsilon)
$$

\section{Proof of Theorem 1.4}

We now proceed with the proof of Theorem 1.4. The construction involves the $(m-1)$-simplex, where $d=2 m-1$. The proof for even dimensions is the same so we only prove the case when $d$ is odd.

Proof Here $d=2 m-1$ and so $k=m+1$. For the first $m$ colour classes, we take

$$
C_{i}=\left\{x \in \mathbb{R}^{d}, x=\left(u_{i}, x e_{i}\right) \in \mathbb{R}^{m-1} \times \mathbb{R}^{m},|x|<\epsilon\right\},
$$


for $i=1, \ldots, m$ and some $\epsilon>0$. Here $u_{i}, i=1, \ldots, m$ are the following vertices of an $(m-1)$-dimensional simplex,

$$
\begin{aligned}
u_{1} & =(1,0,0, \ldots, 0), \\
u_{2} & =-u_{1}=(-1,0,0, \ldots, 0), \\
u_{3} & =(0,1,0, \ldots, 0) \\
& \vdots \\
u_{m} & =(0,0,0, \ldots, 1) .
\end{aligned}
$$

The set $\left\{e_{i}, i=1, \ldots, m\right\}$ is the usual basis of $\mathbb{R}^{m}$. Therefore, for each $i=1, \ldots, m$ the colour class $C_{i}$ is defined as small segment in the direction of $e_{i}$, one for each of the $m$ remaining dimensions. The final colour class will be a small segment at the centre of gravity of the simplex, $\left(0, \frac{1}{m}, \ldots, \frac{1}{m}, 0, \ldots, 0\right)$.

We will show that with this choice of the $u_{i}$ 's, for any transversal, $Y=\left\{y_{i} \in C_{i}\right.$ : $i=1, \ldots, m+1\}$, there is a ball $B$, centred at $\left(u_{0}, v_{0}\right) \in \mathbb{R}^{d}$ of radius $R$ (both to be specified), such that $B \cap C_{i}=\left\{y_{i}\right\}$, for $i=1, \ldots, m$ and $C_{m+1} \subset B$.

Write $u_{0}=\left(u_{0}(1), \ldots, u_{0}(m-1)\right) \in \mathbb{R}^{m-1}, v_{0}=\left(v_{0}(1), \ldots, v_{0}(m)\right) \in \mathbb{R}^{m}$ and $y_{i}=\left(u_{i}, x_{i} e_{i}\right)$. The ball $B$ with the desired properties must satisfy,

$$
\begin{gathered}
\left(\left(u_{0}, v_{0}\right)-\left(u_{i}, x_{i} e_{i}\right)\right) \cdot\left(0, e_{i}\right)=0 \\
\left\|u_{0}-u_{i}\right\|^{2}+\left\|v_{0}-x_{i} e_{i}\right\|^{2}=R^{2},
\end{gathered}
$$

for $i=1, \ldots, m$. Equation (6) shows that each $y_{i}$ lies on the surface of the ball and (5), that each colour class is tangent to the ball at the point $y_{i}$. (Note that here we use the same notation $\|\cdot\|$ for the Euclidean norm in $\mathbb{R}^{m-1}, \mathbb{R}^{m}$ and $\mathbb{R}^{d}$.) From the $m$ equations (5) we get $v_{0}(i)=x_{i}$, for all $i=1, \ldots, m$. Using this, the $m$ equations of (6) can be rewritten as,

$$
\begin{gathered}
\left\|u_{0}-u_{i}\right\|^{2}+\sum_{j \neq i} x_{j}^{2}=R^{2}, \quad i=1, \ldots, m, \quad \text { or } \\
\left\|u_{0}-u_{i}\right\|^{2}+\left\|v_{0}\right\|^{2}=R^{2}+x_{i}^{2}, \quad i=1, \ldots, m .
\end{gathered}
$$

The first two equations of (7) give

$$
u_{0} \cdot u_{1}=\frac{x_{2}^{2}-x_{1}^{2}}{4}
$$

Since $u_{1}=(1,0, \ldots, 0)$, this yields $u_{0}(1)=\frac{x_{2}^{2}-x_{1}^{2}}{4}$. From the first and $(i)$-th equation in (7), for $i=3, \ldots, m$ we get

$$
u_{0} \cdot u_{i}=\frac{x_{1}^{2}-x_{i}^{2}}{2}+u_{0} \cdot u_{1},
$$


where we also used the fact that $\left\|u_{i}\right\|=1$, for all $i=1, \ldots, m$. From this, using (8) we get,

$$
u_{0} \cdot u_{i}=\frac{x_{1}^{2}+x_{2}^{2}-2 x_{i}^{2}}{4}, \quad i=3, \ldots, m .
$$

Since $u_{0} \cdot u_{i}=u_{0}(i-1)$, for $i=3, \ldots, m$ we obtain the remaining coordinates of $u_{0}$,

$$
u_{0}(i-1)=\frac{x_{1}^{2}+x_{2}^{2}-2 x_{i}^{2}}{4}, \quad i=3, \ldots, m .
$$

To specify the radius $R$, we may use any one of the equations from (7). Using say the first, we get

$$
R=\sqrt{\sum_{i=3}^{m} \frac{\left(x_{1}^{2}+x_{2}^{2}-2 x_{i}^{2}\right)^{2}}{16}+\sum_{i=2}^{m} x_{i}^{2}+\frac{\left(x_{2}^{2}-x_{1}^{2}\right)^{2}}{16}-\frac{x_{2}^{2}-x_{1}^{2}}{2}+1}
$$

Hence, for any choice of transversal $Y=\left\{y_{i}, i=1, \ldots, m+1\right\}$, the ball $B$, centred at

$$
\left(u_{0}, v_{0}\right)=\left(\frac{x_{2}^{2}-x_{1}^{2}}{4}, \frac{x_{1}^{2}+x_{2}^{2}-2 x_{3}^{2}}{4}, \ldots, \frac{x_{1}^{2}+x_{2}^{2}-2 x_{m}^{2}}{4}, x_{1}, x_{2}, \ldots, x_{m}\right)
$$

of radius $R$, satisfies (5) and (6). That is, it is tangent to the intervals (colour classes) $C_{i}$ at the points $y_{i}$, for $i=1, \ldots, m$ and $C_{m+1} \subset B$. This completes the proof.

\section{General families}

The result of Theorem 1.1 can be generalized from the family of Euclidean balls to families of subsets defined by a family of functions. Given $\left\{f_{i}: \mathbb{R}^{d} \rightarrow \mathbb{R}, i=\right.$ $1, \ldots, m\}$, let $\mathcal{F} \subset \mathcal{P}\left(\mathbb{R}^{d}\right)$ be the family of sets of the form

$$
F=\left\{x \in \mathbb{R}^{d}: \sum_{i=1}^{m} \alpha_{i} f_{i}(x) \leq 0\right\}
$$

for some $\alpha_{1}, \ldots, \alpha_{m} \in \mathbb{R}$ not all zero. If $T: \mathbb{R}^{d} \rightarrow \mathbb{R}^{m}$ is the map defined by $T(x)=\left(f_{1}(x), \ldots, f_{m}(x)\right)$, then a typical set $F$ is given by $T(x) \cdot a \leq 0$, for some $a=\left(\alpha_{1}, \ldots, \alpha_{m}\right) \in \mathbb{R}^{m}, a \neq 0$. Thus $\mathcal{F}$ is induced by the halfspaces in $\mathbb{R}^{m}$ whose bounding hyperplane passes through the origin.

In this case, Lemma 1.2 and Theorem 1.1 are formulated as follows.

Lemma 5.1 Let $X \subset \mathbb{R}^{d}$, be a finite set, $X=X_{1} \cup X_{2} \cup \cdots \cup X_{k}$, with $X_{i} \cap X_{j}=\varnothing$, for all $i \neq j$, where $k=\left\lfloor\frac{m+3}{2}\right\rfloor$ and $\left|X_{j}\right|=3$. Then, there is a transversal $Y \subset X$ such that for any $F \in \mathcal{F}$ with $Y \subset F$, we have $F \cap(X \backslash Y) \neq \varnothing$.

Theorem 5.2 Let $\mathcal{F}$ be the family of sets in $\mathbb{R}^{d}$ generated by a family of $m$ functions as in (13). Then there is a positive constant $c(m)>0$ such that for any system 
$X_{1}, X_{2}, \ldots, X_{k}$, in $\mathbb{R}^{d}$ with $k=\left\lfloor\frac{m+3}{2}\right\rfloor$ there is a transversal $Y \subset X$, with the property that for any $F \in \mathcal{F}$ with $Y \subset F$, it follows $|F \cap X| \geq c(m)|X|$.

We only prove Lemma 5.1 as the counting argument for Theorem 5.2 is the same as for Theorem 1.1.

Proof Consider the points $T(x) \in \mathbb{R}^{m}, x \in X$. These are divided in $k$ classes $T\left(X_{j}\right), j=1, \ldots, k$ inherited from $\mathbb{R}^{d}$, with $\left|C\left(X_{j}\right)\right|=\left|X_{j}\right|=3, j=1, \ldots, k$, with $k=\left\lfloor\frac{m+3}{2}\right\rfloor$. From Theorem 1 , there are $A_{1}, A_{2} \subset \mathbb{R}^{m}$, with $A_{1} \cap A_{2}=\varnothing$, $\left|A_{i} \cap T\left(X_{j}\right)\right|=1$, for all $i, j$ and conv $A_{1} \cap \operatorname{conv} A_{2} \neq \varnothing$.

Write $A_{1}=\left\{T\left(x_{1}\right), \ldots, T\left(x_{k}\right)\right\}, A_{2}=\left\{T\left(y_{1}\right), \ldots, T\left(y_{k}\right)\right\}$, where $x_{j}, y_{j} \in X_{j}$, $j=1, \ldots, k$. Let $Y_{1}=\left\{x_{1}, \ldots, x_{k}\right\} \subset X$ and $Y_{2}=\left\{y_{1}, \ldots, y_{k}\right\} \subset X$. We claim that either $Y_{1}$ or $Y_{2}$ is the set $Y$ in the statement of the Lemma. That is, either for any $F \in \mathcal{F}$ that contains $Y_{1}$, we have $F \cap Y_{2} \neq \varnothing$ (and therefore $F$ contains a point of $X$ ), or, for any $F \in \mathcal{F}$ that contains $Y_{2}$, we have $F \cap Y_{1} \neq \varnothing$. Indeed, suppose both assertions are false. Then there are $F_{1}, F_{2} \in \mathcal{F}$,

$$
F_{1}=\left\{x \in \mathbb{R}^{d}: \sum_{i=1}^{m} \alpha_{i} f_{i}(x) \leq 0\right\}, \quad F_{2}=\left\{x \in \mathbb{R}^{d}: \sum_{i=1}^{m} \beta_{i} f_{i}(x) \leq 0\right\},
$$

for some $\alpha_{1}, \ldots, \alpha_{m} \in \mathbb{R}$ not all zero, $\beta_{1}, \ldots, \beta_{m} \in \mathbb{R}$ not all zero, such that $Y_{1} \subset F_{1}, Y_{2} \cap F_{1}=\varnothing$ and $Y_{2} \subset F_{2}, Y_{1} \cap F_{2}=\varnothing$. This means that there are $a=$ $\left(\alpha_{1}, \ldots, \alpha_{m}\right) \in \mathbb{R}^{m}, b=\left(\beta_{1}, \ldots, \beta_{m}\right) \in \mathbb{R}^{m}, a, b \neq 0$, such that all of the following hold:

$$
\begin{array}{lll}
a \cdot T\left(x_{j}\right) \leq 0, \quad \forall x_{j} \in Y_{1} \quad \text { and } \quad a \cdot T\left(y_{j}\right)>0, & \forall y_{j} \in Y_{2}, \\
b \cdot T\left(y_{j}\right) \leq 0, \quad \forall y_{j} \in Y_{2} & \text { and } \quad b \cdot T\left(x_{j}\right)>0, & \forall x_{j} \in Y_{1} .
\end{array}
$$

Combining these we get

$$
(a-b) \cdot T\left(x_{j}\right)<0, \quad \forall x_{j} \in Y_{1}
$$

and

$$
(b-a) \cdot T\left(y_{j}\right)<0, \quad \forall y_{j} \in Y_{2} .
$$

Let $w \in \operatorname{conv} A_{1} \cap \operatorname{conv} A_{2}$. Then

$$
\begin{aligned}
w & =\sum_{T\left(x_{j}\right) \in A_{1}} \lambda\left(x_{j}\right) T\left(x_{j}\right)=\sum_{x_{j} \in Y_{1}} \lambda\left(x_{j}\right) T\left(x_{j}\right) \\
& =\sum_{T\left(y_{j}\right) \in A_{2}} \mu\left(y_{j}\right) T\left(y_{j}\right)=\sum_{y_{j} \in Y_{2}} \mu\left(y_{j}\right) T\left(y_{j}\right),
\end{aligned}
$$

for some $0 \leq \lambda\left(x_{j}\right), \mu\left(y_{j}\right) \leq 1, j=1, \ldots, k, \sum_{x_{j} \in Y_{1}} \lambda\left(x_{j}\right)=\sum_{y_{j} \in Y_{2}} \mu\left(y_{j}\right)=1$.

Multiply (14) by $\lambda\left(x_{j}\right) \geq 0, x_{j} \in Y_{1}$ and sum over all $x_{j} \in Y_{1}$ and (15) by $\mu\left(y_{j}\right) \geq 0, y_{j} \in Y_{2}$ and sum over all $y_{j} \in Y_{2}$. Then we get 


$$
\begin{aligned}
0 & >(a-b) \cdot \sum_{x_{j} \in Y_{1}} \lambda\left(x_{j}\right) T\left(x_{j}\right)=(a-b) \cdot w=(a-b) \cdot \sum_{y_{j} \in Y_{2}} \mu\left(y_{j}\right) T\left(y_{j}\right) \\
& =-(b-a) \cdot \sum_{y_{j} \in Y_{2}} \mu\left(y_{j}\right) T\left(y_{j}\right)>0 .
\end{aligned}
$$

This contradiction completes the proof.

Remark 1 The number of color classes in Theorem 5.2 can be improved under certain assumptions on the functions $f_{i}$. We may assume that $f_{m}=1$, since the set $\left\{f_{m}(x), x \in X\right\}$ takes positive, negative or 0 values for at least a third of the points of $X$. If it is 0 , then $T$ maps $X$ to a subspace of dimension $m-1$. If it is positive (or negative) and we replace the functions $f_{i}$ by $f_{i} / f_{m}$ we can map $X$ to an affine subspace of dimension $m-1$. The same is true if we consider sets of the form

$$
F=\left\{x \in \mathbb{R}^{d}: \sum_{i=1}^{m-1} \alpha_{i} f_{i}(x) \leq \alpha\right\},
$$

i.e the sets induced by all halfspaces in $\mathbb{R}^{m-1}$. In this case the number of color classes required is $\left\lfloor\frac{m+2}{2}\right\rfloor$.

Remark 2 Let $X \subset \mathbb{R}^{d}$ be a finite set and $\mathcal{F} \subset \mathcal{P}\left(\mathbb{R}^{d}\right)$ the family defined by the following $d+2$ functions $f_{i}: \mathbb{R}^{d} \rightarrow \mathbb{R}$.

$$
\begin{array}{ll}
f_{i}: & \left(x_{1}, \ldots, x_{d}\right) \mapsto x_{i}, \quad \text { for } i=1, \ldots, d, \\
f_{d+1}: & \left(x_{1}, \ldots, x_{d}\right) \mapsto x_{1}^{2}+\cdots+x_{d}^{2}, \\
f_{d+2}: & \left(x_{1}, \ldots, x_{d}\right) \mapsto 1 .
\end{array}
$$

Clearly $\mathcal{F}$ contains the family of Euclidean balls and their complements, which we denote by $\mathcal{B}_{c}$. According to Theorem 1.1 , if $X$ is partitioned into $\left\lfloor\frac{d+3}{2}\right\rfloor$ color classes, we can find $Y \subset X$ that intersects each color class in a single point and so that if $B \in \mathcal{B}$ contains $Y$, then $B$ contains a positive fraction of the points of $X$. Since $\mathcal{B} \subset \mathcal{F}$, according to Theorem 5.2 (and Remark 1) the number of color classes required to partition $X$ is $\left\lfloor\frac{d+4}{2}\right\rfloor$ (which, in the case where $d$ is even, is one more than the best possible according to Theorems 1.1 and 1.3). It is easy to see that the proofs of Theorem 1.1 and Lemma 1.2 apply to the case of $\mathcal{B}_{c}$ as well. But if a family contains both the balls and their complements, $\left\lfloor\frac{d+3}{2}\right\rfloor$ color classes do not suffice. What fails to be true in this case is Lemma 1.2. For example, in the case $d=2$, any 6 point set $X$ with 3 points colored red and 3 blue, must have two rainbow subsets $A_{1}=\left\{r_{1}, b_{1}\right\}$ and $A_{2}=\left\{r_{2}, b_{2}\right\}$ such that the segments $S_{1}=\operatorname{conv}\left\{r_{1}, b_{1}\right\}, S_{2}=\operatorname{conv}\left\{r_{2}, b_{2}\right\}$ intersect. Clearly the points $r_{1}, r_{2}, b_{1}, b_{2}$ form the vertices of a convex quadrilateral $\Pi$ with diagonals $S_{1}, S_{2}$. It is a well known result that one of the diagonals $S_{1}, S_{2}$ has the property that any disc that contains it, contains at least another vertex of $\Pi$ (see for example [6]). Assume that it is the longest one and let it be $S_{2}$. Then $S_{1}$ has the same property, for the case of the complements of discs. Now choose two discs $B_{1} \subset B_{2}$, with $S_{1} \subset B_{1}$ and $S_{2} \subset B_{2}^{c}$. Then, for the family $\mathcal{F}$ there are $F_{1}=B_{1} \in \mathcal{F}$ and $F_{2}=B_{2}^{c} \in \mathcal{F}$ such that $A_{1} \subset F_{1}, A_{2} \subset F_{2}$ and $A_{1} \cap F_{2}=A_{2} \cap F_{1}=\varnothing$. 
Acknowledgements The author is grateful to Prof. Imre Bárány and Prof. Keith Ball for their encouragement and many suggestions and comments during the preparation of this paper. The author also wishes to thank the UCL Graduate School for awarding the Research Grant, \#1.03/04.RP.EMB and supporting the visit to the Mathematical Sciences Research Institute, Berkeley where most of the preparation of this paper took place. Finally the author wishes to thank MSRI for their hospitality.

\section{References}

1. Bárány, I., Larman, D.G.: A combinatorial property of points and ellipsoids. Discrete Comput. Geom. 5, 375-382 (1990)

2. Bárány, I., Schmerl, J.H., Sidney, S.J., Urrutia, J.: A combinatorial result about points and balls in euclidean space. Discrete Comput. Geom. 4, 259-262 (1989)

3. Hayward, R.: A note on the circle containment problem. Discrete Comput. Geom. 4, 263-264 (1989)

4. Hayward, R., Rappaport, D., Wenger, R.: Some extremal results on circles containing points. Discrete Comput. Geom. 4, 253-258 (1989)

5. Matoušek, J.: Lectures on Discrete Geometry. Springer, Berlin (2002)

6. Neumann-Lara, V., Urrutia, J.: A combinatorial result on points and circles on the plane. Discrete Math. 69, 173-178 (1988)

7. Vrećica, S.T., Živaljević, R.T.: New cases of the colored Tverberg theorem. In: Barcelo, H., Kalai, G. (eds.) Jerusalem Combinatorics '93. Contemporary Mathematics, vol. 178. AMS, Providence (1994)

8. Živaljević, R.T., Vrećica, S.T.: The colored Tverberg's problem and complexes of injective functions. J. Comb. Theory Ser. A 61, 309-318 (1992)

9. Živaljević, R.T.: Topological methods. In: Goodman, J.E., O'Rourke, J. (eds.) Handbook of Discrete and Computational Geometry, new edn. CRC Press, Boca Raton (2004) 